\title{
A new efficient method for solving quadratic Riccati differential equation
}

\author{
Belal Batiha \\ Higher Colleges of Technology (HCT), Abu Dhabi Men's College, United Arab Emirates (UAE) \\ E-mail: belalbatiha2002@yahoo.com, belal.batiha@hct.ac.ae
}

Copyright (C)2015 Belal Batiha. This is an open access article distributed under the Creative Commons Attribution License, which permits unrestricted use, distribution, and reproduction in any medium, provided the original work is properly cited.

\begin{abstract}
A new efficient method called the multistage variational iteration method (MVIM) is applied to the solution of quadratic Riccati differential equations. A comparison between MVIM solution with classical variational iteration method (VIM) and exact solution has been made and show that the MVIM is a powerful method to the solution of nonlinear differential equations.
\end{abstract}

Keywords: Quadratic Riccati equation; Variational iteration method; Multistage variational iteration method.

\section{Introduction}

The Riccati differential equation is named after the Italian nobleman Count Jacopo Francesco Riccati (16761754). The book of Reid [1] contains the fundamental theories of Riccati equation, with applications to random processes, optimal control, and diffusion problems. Besides important engineering science applications that today are considered classical, such as stochastic realization theory, optimal control, robust stabilization, and network synthesis, the newer applications include such areas as financial mathematics [2, 3]. The solution of this equation can be reached using classical numerical methods such as the forward Euler method and Runge-Kutta method. An unconditionally stable scheme was presented by Dubois and Saidi [4]. El-Tawil [5] presented the usage of Adomian decomposition method (ADM) to solve the nonlinear Riccati in an analytic form. Recently, Tan and Abbasbandy [6] employed the analytic technique called Homotopy Analysis Method (HAM) to solve a quadratic Riccati equation. Very recently, Geng [7] presented a modified variational iteration method to solve quadratic Riccati equation.

The variational iteration method (VIM) is a simple and yet powerful method for solving a wide class of nonlinear problems, first envisioned by He [8] (see also [9, 10, 11, 12, 13, 14, 15, 16, 17]). The VIM has successfully been applied to many situations. For example, He [9] solved the classical Blasius' equation using VIM. He [10] gave a solution for seepage flow with fractional derivatives in porous media using VIM. He [11] used VIM to give approximate solutions for some well-known non-linear problems. He [12] used VIM to solve autonomous ordinary differential systems. He [13] coupled the iteration method with the perturbation method to solve the well-known Blasius equation. He [15] solved strongly nonlinear equations using VIM. Soliman [18] applied the VIM to solve the KdV-Burger's and Lax's seventh-order KdV equations. The VIM has recently been applied for solving nonlinear coagulation problem with mass loss by Abulwafa [19]. Momani [20] applied VIM to Helmholtz equation. The VIM has been applied for solving nonlinear differential equations of fractional order by Odibat [21]. Bildik [22] used VIM for solving different types of nonlinear partial differential equations. Dehghan and Tatari [23] employed VIM to solve a Fokker-Planck equation. Wazwaz [24] used VIM to determine rational solutions for the KdV, the $K(2,2)$, the Burgers, and the 
cubic Boussinesq equations. Tamer [25] introduced a modification of VIM. Abbasbandy [26] solved the quadratic Riccati differential equation by He's variational iteration method with considering Adomian's polynomials. Junfeng [27] applied VIM to solve two-point boundary value problems.

This paper centralized on modified version of VIM, which is called multistage variational iteration method (MVIM) and we will present comparative solutions with VIM and exact solution. The MVIM speeds the convergence of the solutions rapidly. It has been applied to many problems such as in [28, 29, 30, 31]. We shall investigate VIM and MVIM accuracy for a longer time frame to show its reliability to Riccati equation.

\section{Variational iteration method}

This method, which is a modified general Lagrange's multiplier method [32], has been shown to solve effectively, easily and accurately a large class of nonlinear problems $[8,9,10,11,12,13,14,15,16,17,18,19,20,21,22,33,34]$. The main feature of the method is that the solution of a mathematical problem with linearization assumption is used as initial approximation or trial function. Then a more highly precise approximation at some special point can be obtained. This approximation converges rapidly to an accurate solution. To illustrate the basic concepts of the VIM, we consider the following nonlinear differential equation:

$L u+N u=g(x)$

where $L$ is a linear operator, $N$ is a nonlinear operator, and $g(x)$ is an inhomogeneous term. According to the VIM $[11,12,13,14,15,16,17]$, we can construct a correction functional as follows:

$u_{n+1}(t)=u_{n}(t)+\int_{0}^{t} \lambda\left\{L u_{n}(s)+N \tilde{u}_{n}(s)-g(s)\right\}$,

where $\lambda$ is a general Lagrangian multiplier [32], which can be identified optimally via the variational theory, the subscript $n$ denotes the $n$ th-order approximation, $\tilde{u}_{n}$ is considered as a restricted variation [11, 12, 13, 14], i.e. $\delta \tilde{u}_{n}=0$.

\section{Multistage variational iteration method (MVIM)}

Multistage variational iteration method or MVIM was first introduced by Batiha et al. [28] on a class of nonlinear system of ordinary differential equations. This methodology was inspired by various researches [35, 36, 37] who used multistage Adomian decomposition method to solve systems of linear and nonlinear equations. It is a technique which allowed a simple modification of the standard VIM. Instead of having the integral contained in the correction functional (2) with a fixed lower limit of 0 , we use a variable $t^{*}$ as a substitute

$u_{n+1}(t)=u_{n}(t)+\int_{t *}^{t} \lambda\left\{L u_{n}(s)+N \tilde{u}_{n}(s)-g(s)\right\}$,

where $t^{*}$ increases according to the designated time-step size in each iteration computation. Each increased timestep will produce a new approximation value for the desired iteration step and the process is continued until the targeted time frame is achieved. The MVIM is more stable compared to the standard VIM [28]. Basically, the initial approximation in each interval is taken from the solution in the previous interval and each new initial approximation is substituted into the subsequent calculation, making it a fast-forwarding kind of solver. Errors are therefore minimized but the disadvantage is having longer computational time. Convergence to accurate solutions depends highly on the choice of initial approximations which then determine the number of iterates which give the most precise solution. Equivalently as important is the size of time steps used in the iterations.

\section{Analysis of Riccati differential equation}

First, we present numerical and analytical solutions for the general Riccati differential equation [38]:

$\frac{d y}{d t}=Q(t) y+R(t) y^{2}+P(t), \quad y(0)=G(t)$ 
where $Q(t), R(t), P(t)$ and $G(t)$ are scalar functions. To solve equation (4) by means of multistage variational iteration method (MVIM), we construct a correction function,

$$
\begin{aligned}
y_{n+1}(t) & =y_{n}(t)+\int_{t^{*}}^{t} \lambda(s)\left[\frac{d y_{n}(s)}{t}-Q(s) \tilde{y}_{n}(s)-R(s) \tilde{y}_{n}^{2}(s)-P(s)\right], \\
\delta y_{n+1}(t) & =\delta y_{n}(t)+\delta \int_{t^{*}}^{t} \lambda(s)\left[\frac{d y_{n}(s)}{t}-Q(s) \tilde{y}_{n}(s)-R(s) \tilde{y}_{n}^{2}(s)-P(s)\right], \\
\delta y_{n+1}(t) & =\delta y_{n}(t)+\delta \int_{t^{*}}^{t} \lambda(s)\left[\frac{d y_{n}(s)}{}\right], \\
\delta y_{n+1}(t) & =(1+\lambda) \delta y_{n}(t)-\int_{t^{*}}^{t} \delta y_{n}(s) \lambda^{\prime}=0,
\end{aligned}
$$

where $\tilde{y}_{n}$ is considered as restricted variations, which mean $\delta \tilde{y}_{n}=0$ and observing that $\delta y_{n}\left(t^{*}\right)=0$ Its stationary conditions can be obtained as follows

$1+\lambda\left(t^{*}\right)=0,\left.\quad \lambda^{\prime}(s)\right|_{s=t^{*}}=0$.

The Lagrange multipliers, therefore, can be identified as $\lambda(s)=-1$ and the following variational iteration formula is obtained

$y_{n+1}(t)=y_{n}(t)-\int_{t^{*}}^{t}\left[\frac{d y_{n}(s)}{}-Q(s) y_{n}(s)-R(s) y_{n}^{2}(s)-P(s)\right]$.

Now we consider the quadratic Riccati differential equation

$\frac{\mathrm{y}}{\mathrm{t}}=2 y(t)-y^{2}(t)+1, \quad y(0)=0$.

Here $Q(t)=2, R(t)=-1, P(t)=1$ and $G(t)=0$.

The exact solution was found to be [5]:

$y(t)=1+\sqrt{2} \tanh \left(\sqrt{2} t+\frac{1}{2} \log \left(\frac{\sqrt{2}-1}{\sqrt{2}+1}\right)\right)$.

To solve equation (6) using MVIM, we construct a correction functional (see (5)),

$y_{n+1}(t)=y_{n}(t)-\int_{t^{*}}^{t}\left[\frac{\mathrm{y}_{n}}{\mathrm{~s}}-2 y_{n}+y_{n}^{2}-1\right]$.

\section{Numerical results and discussion}

The MVIM algorithm is coded in the computer algebra package Maple. The Maple environment variable Digits controlling the number of significant digits is set to 16 in all the calculations done in this paper. We now obtain numerical solutions of quadratic Riccati differential equation. In table 1, we compare MVIM against the standard VIM [39] and the exact solution for $t \in[0,20]$. We choose to apply MVIM using only two iterations and step size $h=0.01$. We found that MVIM is far more stable than the three iteration of VIM in the case investigated. Table 2 shows a detailed view of the solutions by zooming into the time range $t=[0,2]$. We can see that the results from classical VIM is near the exact results just in a very short time frame $t=[0,1.4]$, after that the results are not reliable at all. 
Table 1: Numerical comparisons for $t=[0,20]$

\begin{tabular}{cccc}
\hline$t$ & Exact solution & 2-Iterate MVIM & 3-Iterate VIM [39] \\
\hline 2 & 2.3577716530 & 2.3592420980 & -286352.73097900 \\
4 & 2.4140123820 & 2.4140330560 & $-9.0657980428 \mathrm{E} 19$ \\
6 & 2.4142128590 & 2.4142130890 & $-7.3332282199 \mathrm{E} 33$ \\
8 & 2.4142135600 & 2.4142136400 & $-5.7930892793 \mathrm{E} 47$ \\
10 & 2.4142135620 & 2.4142136400 & $-4.5744317440 \mathrm{E} 61$ \\
12 & 2.4142135620 & 2.4142136400 & $-3.6121072512 \mathrm{E} 75$ \\
14 & 2.4142135620 & 2.4142136560 & $-2.8522268182 \mathrm{E} 89$ \\
16 & 2.4142135620 & 2.4142136940 & $-2.2522027269 \mathrm{E} 103$ \\
18 & 2.4142135620 & 2.4142136830 & $-1.7784059425 \mathrm{E} 117$ \\
20 & 2.4142135620 & 2.4142136830 & $-1.4042819762 \mathrm{E} 131$ \\
\hline
\end{tabular}

Table 2: Numerical comparisons for $t=[0,2]$

\begin{tabular}{cccc}
\hline$t$ & Exact solution & 2-Iterate MVIM & 3-Iterate VIM [39] \\
\hline 0.2 & 0.2419767992 & 0.2396149017 & 0.2419778327 \\
0.4 & 0.5678121656 & 0.5626231618 & 0.5678455132 \\
0.6 & 0.9535662155 & 0.9468409011 & 0.9536660329 \\
0.8 & 1.3463636550 & 1.3405640980 & 1.3463791062 \\
1.0 & 1.6894983900 & 1.6863821450 & 1.6860271032 \\
1.2 & 1.9513601180 & 1.9509491870 & 1.9150510260 \\
1.4 & 2.1313266100 & 2.1325827440 & 2.1791315021 \\
1.6 & 2.2462859590 & 2.2481414290 & -50.98229780 \\
1.8 & 2.3163247370 & 2.3181237490 & -5338.782860 \\
2.0 & 2.3577716530 & 2.3592420980 & -286352.7325 \\
\hline
\end{tabular}

\section{Conclusions}

In this paper, multistage variation iteration method (MVIM) has been successfully applied to find the approximate solution of the quadratic Riccati differential equation. The method was used in a direct way without using linearization, perturbation or restrictive assumptions. Therefore, it is not affected by computation round off errors and one is not faced with necessity of large computer memory and time. This method unlike most numerical techniques provides a closed-form solution. A specific advantage of this method over any purely numerical method is that it offers a smooth, functional form of the solution over a time step. Moreover, the present procedure offers an explicit timemarching algorithm that works accurately over such high step sizes for which most of the available integration schemes will fail to be accurate. Based on the cases investigated, MVIM is more stable for a longer timespan than the standard VIM. It may be concluded that MVIM is very powerful and efficient in finding analytical as well as numerical solutions for wide classes of linear and nonlinear differential equations. It provides more realistic series solutions that converge very rapidly in real physical problems due to its consistency used in a longer time frame.

\section{References}

[1] Reid WT. Riccati differential equations (Mathematics in science and engineering, Vol. 86). New York: Academic Press.; 1972 .

[2] Anderson BD, Moore JB. Optimal control-linear quadratic methods. Prentice-Hall, New Jersey 1999.

[3] Lasiecka I, Triggiani R. Differential and algebraic Riccati equations with application to boundary/point control problems: continuous theory and approximation theory (Lecture notes in control and information sciences, Vol. 164). Berlin: Springer.; 1991. 
[4] Dubois F, Saidi A. Unconditionally stable scheme for Riccati equation. ESAIM Proceedings 8 (2000) 39-52.

[5] Bahnasawi AA, El-Tawil MA, Abdel-Naby A. Solving Riccati differential equation using Adomian's decomposition method. Appl Math Comput 157 (2004) 503-514.

[6] Tan Y, Abbasbandy S. Homotopy analysis method for quadratic Riccati differential equation. Commun Nonlin Sci Numer Simul 13 (3) (2008) 539-546.

[7] Geng, F. A modified variational iteration method for solving Riccatic differential equations. Comp.Math. with Application 6 (2010) 1868-1872.

[8] He JH. A new approach to nonlinear partial differential equations. Commun Nonlin Sci Numer Simul 2 (1997) $230-235$.

[9] He JH. Approximate analytical solution of Blasius' equation. Commun Nonlin Sci Numer Simul 3 (1998) $260-263$.

[10] He JH. Approximate analytical solution for seepage flow with fractional derivatives in porous media. Comput Meth Appl Mech Engrg 167 (1998) 57-68.

[11] He JH. Variational iteration method-a kind of non-linear analytical technique: some examples. Int J Non-Linear Mech 34 (1999) 699-708.

[12] He JH. Variational iteration method for autonomous ordinary differential systems. Appl Math Comput 114 (2000) 115-123.

[13] He JH. A simple perturbation approach to Blasius equation. Appl Math Comput 140 (2003) 217-222.

[14] He JH, Wan YQ, Guo Q. An iteration formulation for normalized diode characteristics. Int J Circ Theory Appl 32 (2004) 629-632.

[15] He JH. Some asymptotic methods for strongly nonlinear equations. Int J Modern Phys B 20 (2006) 1141-1199.

[16] He JH. Non-perturbative methods for strongly nonlinear problems. Berlin: dissertation.de-Verlag im Internet GmbH.; 2006.

[17] He JH. Variational iteration method-some recent results and new interpretations. J Comput Appl Math 207 (1) (2007) $3-17$.

[18] Soliman AA. A numerical simulation and explicit solutions of KdV-Burgers' and Lax's seventh-order KdV equations. Chaos Solitons Fractals 29 (2) (2006) 294-302

[19] Abulwafa EM, Abdou MA, Mahmoud AA. The solution of nonlinear coagulation problem with mass loss. Chaos Solitons Fractals 29 (2) (2006) 313-330.

[20] Momani S, Abuasad S. Application of He's variational iteration method to helmholtz equation. Chaos Solitons Fractals 27 (2006) 1119-1123.

[21] Odibat ZM, Momani S. Application of variational iteration method to nonlinear differential equations of fractional order. Int J Nonlinear Sci Numer Simul 7 (2006) 27-34.

[22] Bildik N, Konuralp A. The use of variational iteration method, differential transform method and Adomian decomposition method for solving different types of nonlinear partial differential equations. Int J Nonlinear Sci Numer Simul 7 (2006) 65-70.

[23] Dehghan M, Tatari M. The use of He's variational iteration method for solving a Fokker-Planck equation. Phys Scr 74 (2006)310-316.

[24] Wazwaz AM. The variational iteration method for rational solutions for $\mathrm{KdV}, \mathrm{K}(2,2)$, Burgers, and cubic Boussinesq equations. J Comput Appl Math 207(1) (2007) 18-23 .

[25] Tamer A. Abassy, Magdy A. El-Tawil, H. El Zoheiry. Toward a modified variational iteration method. J Comput Appl Math. 207(1) (2007) 137-147 .

[26] Abbasbandy S. A new application of He's variational iteration method for quadratic Riccati differential equation by using Adomian's polynomials. J Comput Appl Math. 207(1) (2007) 59-63. .

[27] Junfeng L. Variational iteration method for solving two-point boundary value problems. J Comput Appl Math. 207(1) (2007) 92-95 . 
[28] Batiha B, Noorani M S M, Hashim I , Ismail E S. The multistage variational iteration method for a class of nonlinear system of ODEs. Phys. Scr. 76 (2007) 388-392

[29] Goh S.M, Noorani M.S.M., Hashim I. Prescribing a multistage analytical method to a preypredator dynamical system. Physics Letters A 373 (2008) 107-110.

[30] Goh S.M, Noorani M.S.M., Hashim I. A new application of variational iteration method for the chaotic Rössler system. Chaos, Solitons and Fractals 42 (2009) 1604-1610.

[31] Goh S.M, Noorani M.S.M., Hashim I. Efficacy of variational iteration method for chaotic Genesio system-Classical and multistage approach. Chaos, Solitons and Fractals 40 (2009) 2152-2159.

[32] Inokuti M. Sekine H, Mura T. General use of the Lagrange multiplier in nonlinear mathematical physics. In: NematNassed S, editor. Variational method in the mechanics of solids. Pergamon Press.; 1978.

[33] Abdou MA, Soliman AA. Variational iteration method for solving Burger's and coupled Burger's equations. J Comput Appl Math 181 (2005) 245-251.

[34] Moghimi M, Hejazi F. Variational iteration method for solving generalized Burger-Fisher and Burger equations. Chaos Solitons Fractals, 33(5) (2007) 1756-1761. .

[35] Abdulaziz O, Noor NFM, Hashim I, Noorani MSM. Further accuracy tests on Adomian decomposition method for chaotic systems. Chaos, Solitons and Fractals 36 (2008) 1405-11.

[36] Hashim I, Noorani MSM, Ahmad R, Bakar SA, Ismail ES, Zakaria AM, et al. Accuracy of the Adomian decomposition method applied to the Lorenz system. Chaos, Solitons and Fractals 28 (2006) 1149-1158.

[37] Noorani MSN, Hashim I, Ahmad R, Bakar SA, Ismail ES, Zakaria AM, et al. Comparing numerical methods for the solutions of the Chen system. Chaos, Solitons and Fractals 32 (4) (2007)1296-1304.

[38] Polyanin AD, Zaitsev VF. Handbook of exact solutions for ordinary differential equations. Florida: CRC.; 2003.

[39] Batiha B, Noorani M S M, Hashim I. Application of variational iteration method to a general Riccati equation. International Mathematical Forum, 2 (56) (2007) 2759-2770. 